\title{
Intellectual Disability, Unusual Facial Morphology and Hand Anomalies in Sibs
}

\section{Sérgio B. Sousa, ${ }^{1,2 *}$ Margarida Venâncio, ${ }^{1}$ Estelle Chanudet, ${ }^{3}$ Rodger Palmer, ${ }^{4}$ Lina Ramos, ${ }^{1}$ Philip L. Beales, ${ }^{5}$ Gudrun E. Moore, ${ }^{2}$ Jorge M. Saraiva, ${ }^{1,6}$ and Raoul C. Hennekam?}

${ }^{1}$ Serviço de Genética Médica, Hospital Pediátrico, Centro Hospitalar e Universitário de Coimbra, Coimbra, Portugal

${ }^{2}$ Clinical and Molecular Genetics Unit, UCL Institute of Child Health, London, UK

${ }^{3}$ GOSgene, UCL Institute of Child Health, London, UK

${ }^{4}$ NE Thames Regional Genetics Service Laboratories, Great Ormond Street Hospital for Children, London, UK

${ }^{5}$ Molecular Medicine Unit, UCL Institute of Child Health, London, UK

${ }^{6}$ University Clinic of Pediatrics, Faculty of Medicine, University of Coimbra, Coimbra, Portugal

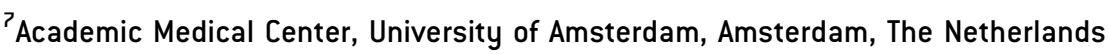

Manuscript Received: 25 April 2013; Manuscript Accepted: 10 June 2013

Here we report on a Portuguese family with three sisters who shared moderate intellectual disability, unusual facial morphology (short palpebral fissures; broad nasal tip; thin upper and lower vermillion; broad and pointed chin) and hand anomalies in two of them (short left third and fifth right metacarpals in one case; marked syndactyly between the third and fourth fingers in another). One of the sisters had microcephaly and short stature, and the other two were obese. Obesity and somewhat similar facial features were also present in the otherwise healthy mother. Despite the overlap with several known syndromes (Albright osteodystrophy; Filippi syndrome; Rubinstein-Taybi syndrome; microdeletion 2q37), we suggest this condition is previously unreported, and most likely displays an autosomal recessive pattern of inheritance. (c) 2013 Wiley Periodicals, Inc.

Key words: intellectual disability; dysmorphisms; brachydactyly; syndactyly; obesity

\section{INTRODUCTION}

Syndromes that are characterized by intellectual disability and hand anomalies constitute a relatively narrow spectrum of disorders [Hennekam et al., 2010]. Here we describe a Portuguese family in which three sisters were affected by a variable combination of these signs and symptoms. We propose the combination in this family represents a hitherto undescribed entity.

\section{CLINICAL REPORTS}

The family originated from the Central Region of Portugal and consisted of three affected sisters and one unaffected brother, born

\section{How to Cite this Article:}

Sousa SB, Venâncio M, Chanudet E, Palmer R, Ramos L, Beales PL, Moore GE, Saraiva JM, Hennekam RC. 2013. Intellectual disability, unusual facial morphology and hand anomalies in sibs.

Am J Med Genet Part A 161A:2401-2406.

to healthy, consanguineous parents (second cousins, once removed). Both parents and brother were of normal intelligence and had a normal height. The mother (Fig. 1) was obese and had facial features somewhat similar to her three daughters. Family history was otherwise unremarkable.

\section{Patient 1}

The eldest sister was born after an uneventful term pregnancy weighing at birth approximately 4,000 g. Motor milestones were attained at a normal age. Main concerns arose when moderate learning difficulties were noticed at school. Her speech and language have always been problematic but were reported to become more delayed between the ages of 5 and 10 years. Menarche occurred at 12 years, menses were regular. She never had major health problems, and hearing and vision were normal.

\footnotetext{
*Correspondence to:
}

Sérgio B. Sousa, Serviço de Genética Médica, Hospital Pediátrico, Av. Afonso Romão, 3000-602 Coimbra, Portugal.

Email: sbsousa@chc.min-saude.pt, sergio.sousa.09@ucl.ac.uk Article first published online in Wiley Online Library (wileyonlinelibrary.com): 15 August 2013

DOI 10.1002/ajmg.a.36124 

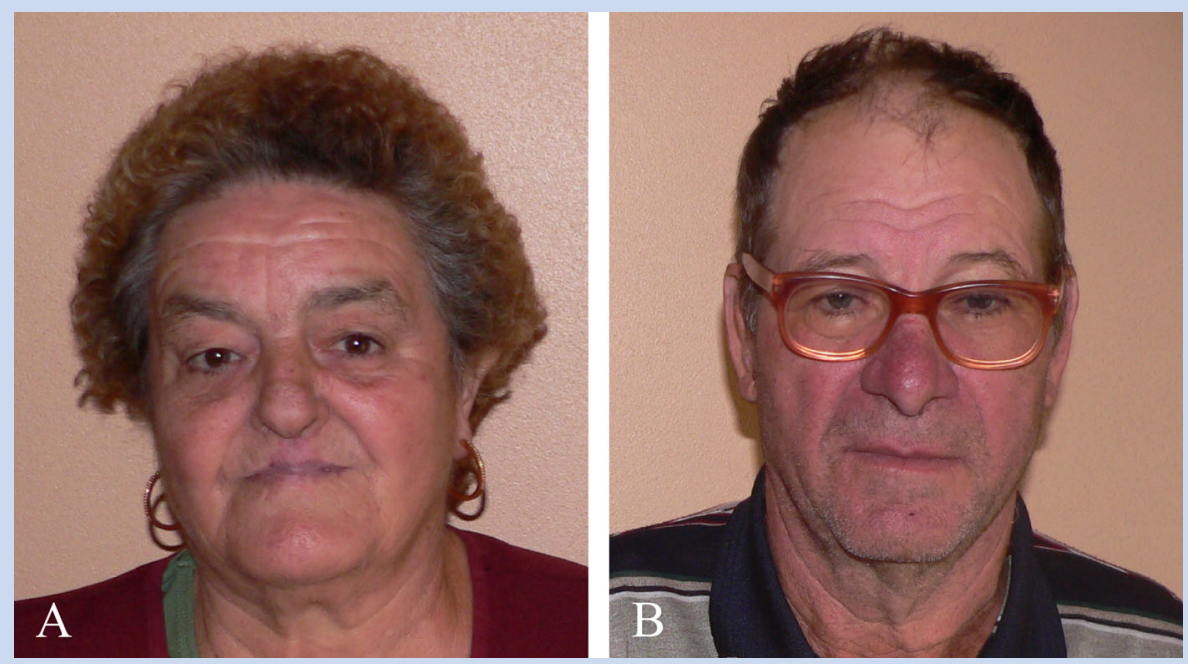

FIG. 1. Healthy and intellectually normal parents. Photographs of mother at 68 years $[A]$ and father at 78 years $(B)$ of age.

At 37 years, a cholecystectomy was performed. When evaluated at 47 years she was estimated to have a moderate intellectual disability (no formal cognitive evaluation had ever been done) and a friendly behavior, though sometimes stubborn and remarkably afraid of hot water. She had simple, limited speech and could not read, write or count. She had short stature (height $1.49 \mathrm{~cm},<5$ th centile, corresponding to the 50 th centile at 11 years and 10 months), microcephaly (OFC $51 \mathrm{~cm},<3 \mathrm{rd}$ centile, corresponding to the 50th centile of 5 years), and normal body weight (54 kg, 10-25th centile, BMI 24.3). She had unusual facial features: short palpebral fissures, broad nasal tip, thin upper and lower vermilions and broad, pointed chin (Fig. 2). She had brachydactyly with especially short distal phalanges, limited flexion in her fingers, marked shortening of the third metacarpal of one hand, a unilateral single palmar crease and abnormal position of the finger creases (Fig. 2D,E). Radiographs of the hands showed marked shortening of the left third and of the right fifth metacarpals (Fig. 2F). Her toes were short (Fig. 2G).

\section{Patient 2}

The second sister was born after an uneventful pregnancy, with unknown birth parameters. Her motor milestones were normal. Similarly to her older sister, she had severe language difficulties, which reportedly became more marked in late childhood. She was considered to have more severe learning difficulties than her two sisters. She had recurrent otitis media in childhood and developed moderate deafness. She walked very carefully and was afraid to walk down stairs and use elevators. At 43 years of age, she was overweight (weight $98 \mathrm{~kg}$, >95th centile; BMI = 33.9), of normal height $(1.70 \mathrm{~m}, 75-90$ th centile), and had normal OFC $(54.5 \mathrm{~cm}$, 50th centile). Her facial features resembled closely those of her sister. Additionally, she had a grimacing smile (Fig. 3) and two hypopig- mented scalp hair patches which had been present since birth. Both hands and feet were normally formed. Radiography could not be performed.

\section{Patient 3}

The youngest sister was born at 40 weeks of gestation after an uneventful pregnancy, weighing approximately 4,000 g. Delivery was difficult and required a vacuum extraction. After birth, she was found to have a right clavicle fracture and a right brachial plexus palsy that improved with physiotherapy. The syndactylies were noticed at birth. She developed moderate language and learning difficulties but otherwise never experienced significant illnesses or hearing and vision problems. At 31 years she had normal stature (height $1.72 \mathrm{~cm}$; 90th centile), normal OFC ( $54.5 \mathrm{~cm}$; 50th centile) and was overweight (weight $104 \mathrm{~kg}$, $>95$ th centile; BMI = 35.2). Her facial morphology was similar to her sisters (Fig. 4). She had right upper limb palsy. No pectoral asymmetry was noted. She had complete syndactyly of the third and fourth fingers, cutaneous at the right side (Fig. 4I,O,Q,S,U) and osseous at the left side (Fig. 4H,N,P,R,T). Her left thumb was slightly short and broad with a very small thumbnail and the distal phalanx was conical in shape and laterally deviated (Fig. 4K). On the X-ray, the left thumb (Fig. 4L,M) had a small terminal phalanx and an extra bone suggesting a partial duplication or triphalangeal thumb. Her toes were short with narrow hyperconvex toenails (Fig. 4J). A full skeletal survey at the age of 32 years demonstrated C2-C3 fusion (Fig. 4G) and no other skeletal abnormalities.

\section{Additional Investigations}

Classical cytogenetic, telomeres FISH and transferrin studies were performed in the youngest sister and gave normal results. 

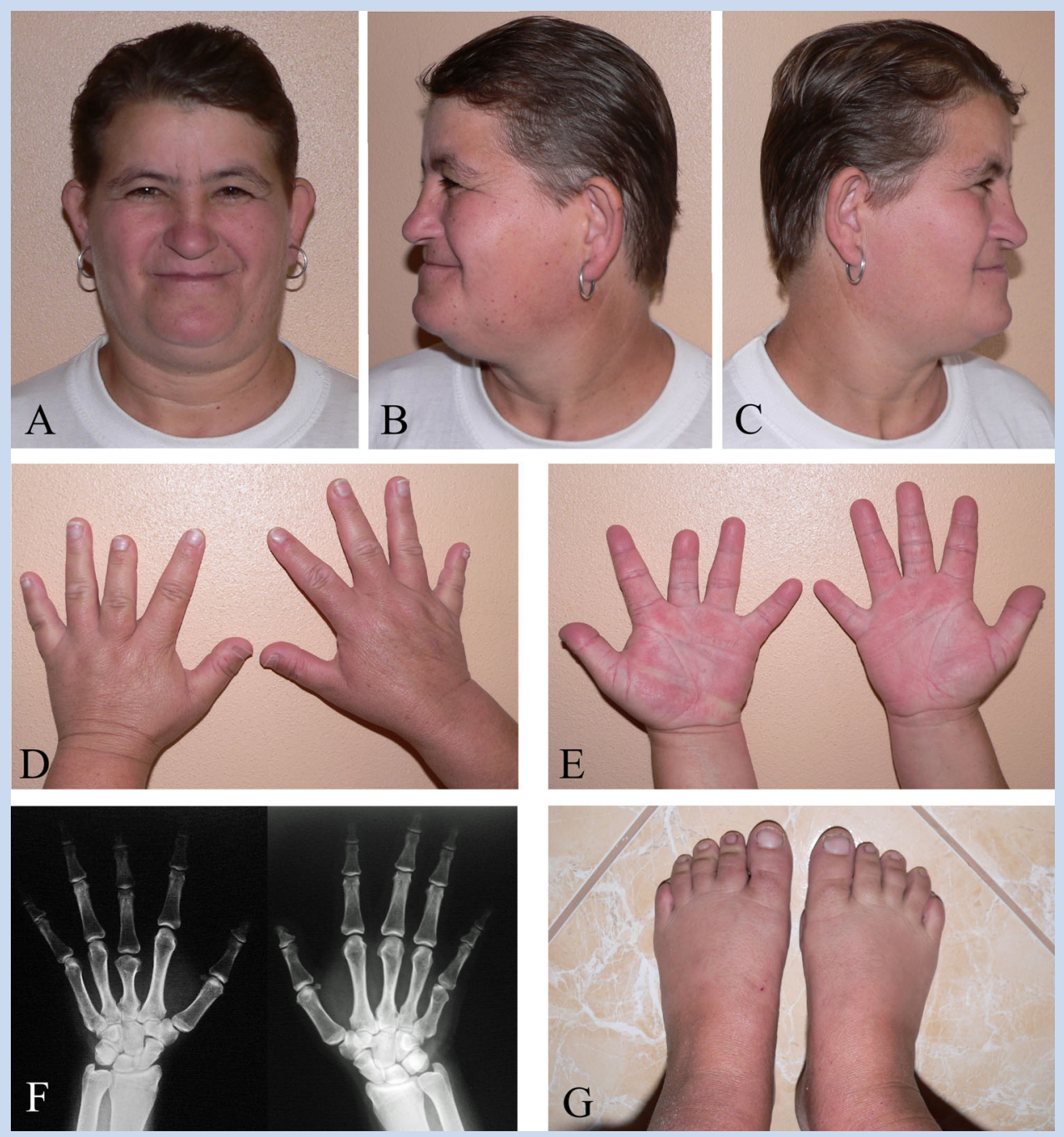

FIG. 2. Eldest sister. Photographs at 47 years of age $(A-E, G]$. Note the short palpebral fissures, broad nasal tip, thin upper and lower vermilions and broad, pointed chin, the left single palmar crease, general brachydactyly, short terminal phalanges, and short toes. Hands radiographs at 48 years of age $[F]$. Note the shortening of the 3rd left and 5 th right metacarpals.

Genome wide array comparative genomic hybridization (aCGH) analysis in the eldest sister using the Nimblegen $720 \mathrm{k}$ array indicated that there was no evidence of any copy number change with clear clinical significance. It identified two copy number variation changes of unknown significance: a heterozygous loss of approximately $10 \mathrm{~Kb}$ at $5 \mathrm{q} 22.1$ which contained no known gene; and a heterozygous gain at band $14 \mathrm{q} 11.2$ which was approximately $0.14 \mathrm{Mb}$ in length and included nine genes (REM2, MIR4707, MMP14, PRMT5, C14orf93, LRP10, HAUS4, RBM23, and JUB). The same $14 \mathrm{q} 11.2$ gain was also identified in the mother, in the two younger sisters and in the unaffected brother using a genome-wide SNP array (Infinium HD HumanCytoSNP-12 BeadChip, Illumina). X-Chromosome inactivation studies were performed in the mother and the three affected sisters and did not show clear skewing: mother-71:29; eldest sister-78:22; middle sister-56:46; younger sister-not informative.

\section{DISCUSSION}

The presently described phenotype in the sisters is characterized by: in all three sisters moderate intellectual disability and unusual facial features (short palpebral fissures, bulbous nasal tip, thin upper and lower vermilions, grimacing smile, pointed chin); and anomalies in two sisters with metacarpal shortening in one and severe 3rd-4th finger syndactyly in another; obesity in two sisters; microcephaly and short stature in one sister. The normally intelligent mother shares the obesity and similar but 

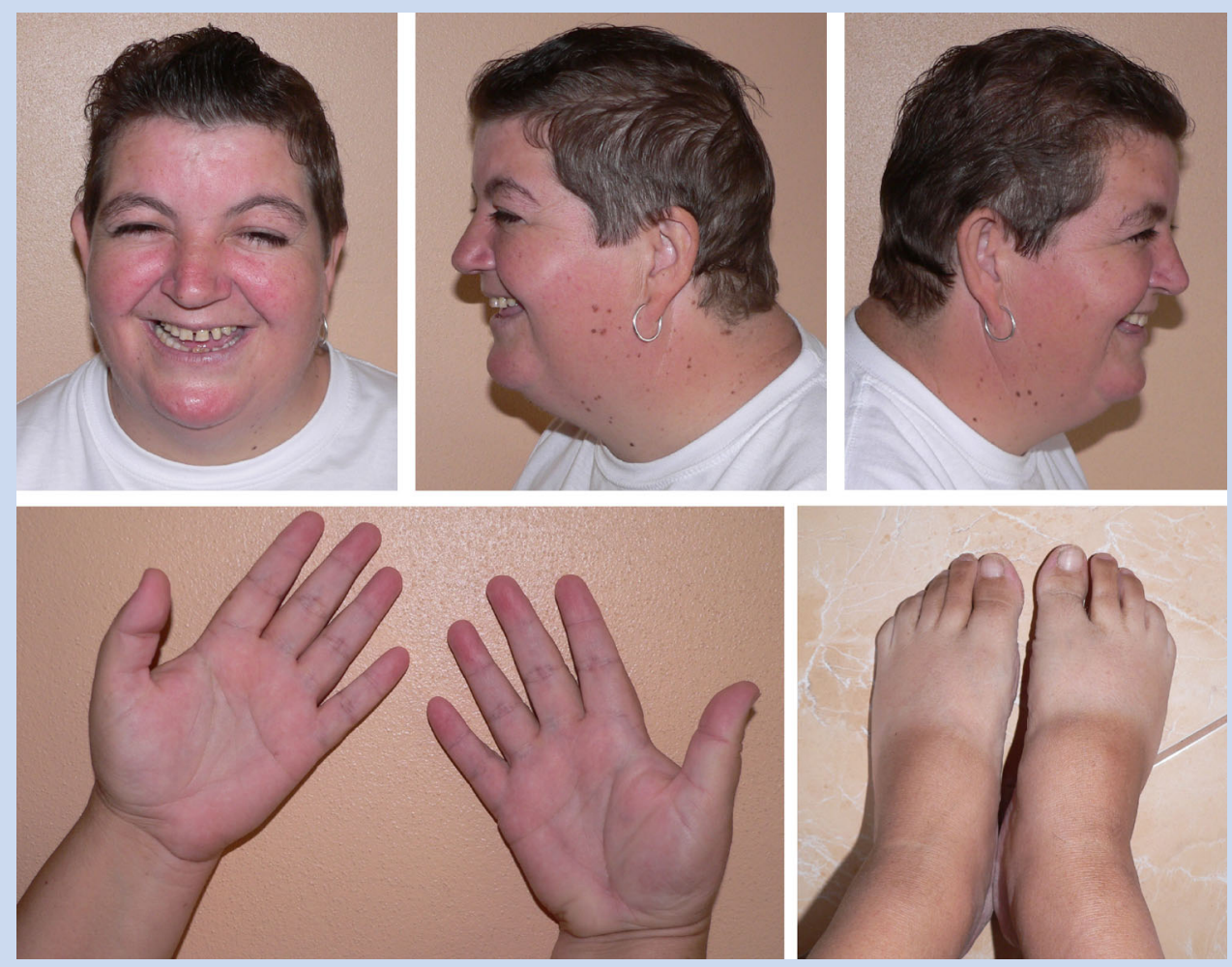

FIG. 3. Middle sister at 43 years of age. Note the resemblance in facial features to those in her sister. In addition she shows a grimacing smile. Hands and feet are normal.

less obvious facial signs (Fig. 1). This may indicate that these signs may not be related to the entity in the sisters. If these features are part of this syndrome (i.e., associated with the intellectual disability and the hands anomalies), one may argue that if this is an autosomal recessive entity, these features may represent manifestations in a heterozygote, and if this is an autosomal dominant entity, it might mean that the phenotype can vary widely also with respect to cognition. We also hypothesized that this condition could be X-linked and its intra-familiar variability explained by lyonization differences. The X-chromosome inactivation studies performed did not support this hypothesis however.

We considered whether the 14q11.2 gain identified could be pathogenic. A much larger, overlapping telomeric duplication (5.38 Mb, including 281 genes) has been described in a male with mild intellectual disability, unusual facial features, and short stature [Monfort et al., 2007]. The latter patient had no hand anomalies, and his facial features showed only very limited resemblance to the present sisters. One may hypothesize that this gain contributed to some of the features of the phenotype, but its presence in the mother and especially also in the healthy brother, who is intellectually normal without obesity and without any facial resemblance to his sisters, makes it unlikely.
The eldest sister shows some similarities with Albright hereditary osteodystrophy (OMIM 103580) but this diagnosis would not explain the hand anomalies in the youngest sister. The eldest sister also shows resemblance to a microdeletion of 2q37 (OMIM 600430), but telomeric FISH studies and the aCGH excluded this. Some facial features resemble Rubinstein-Taybi syndrome (OMIM 180849) and also the behavior of the sisters would fit in, but the distal limb anomalies, growth pattern, and familial occurrence argue strongly against this diagnosis. Filippi [1985] syndrome (OMIM 272440) is characterized by microcephaly, syndactyly of both hands and feet, and intellectual disability with prominent weakness in speech and communication. The syndactyly in Filippi syndrome is most frequently between the 3rd and 4th fingers, similar to the syndactyly in the youngest sister, but is typically only cutaneous. Furthermore, the absence of syndactyly between the toes in any of the present patients and the difference in facial characteristics allows us to differentiate the entities. Other possible disorders to consider in the differential diagnosis have been Feingold syndrome [Feingold et al., 1997] (OMIM 164280), oculodentodigital dysplasia [Gorlin et al., 1963] (OMIM 164200), Tsukahara syndrome [Tsukahara et al., 1989] (OMIM 613627), Tonoki syndrome [Tonoki et al., 1990] (OMIM 603396) and the patient reported by Ravel 

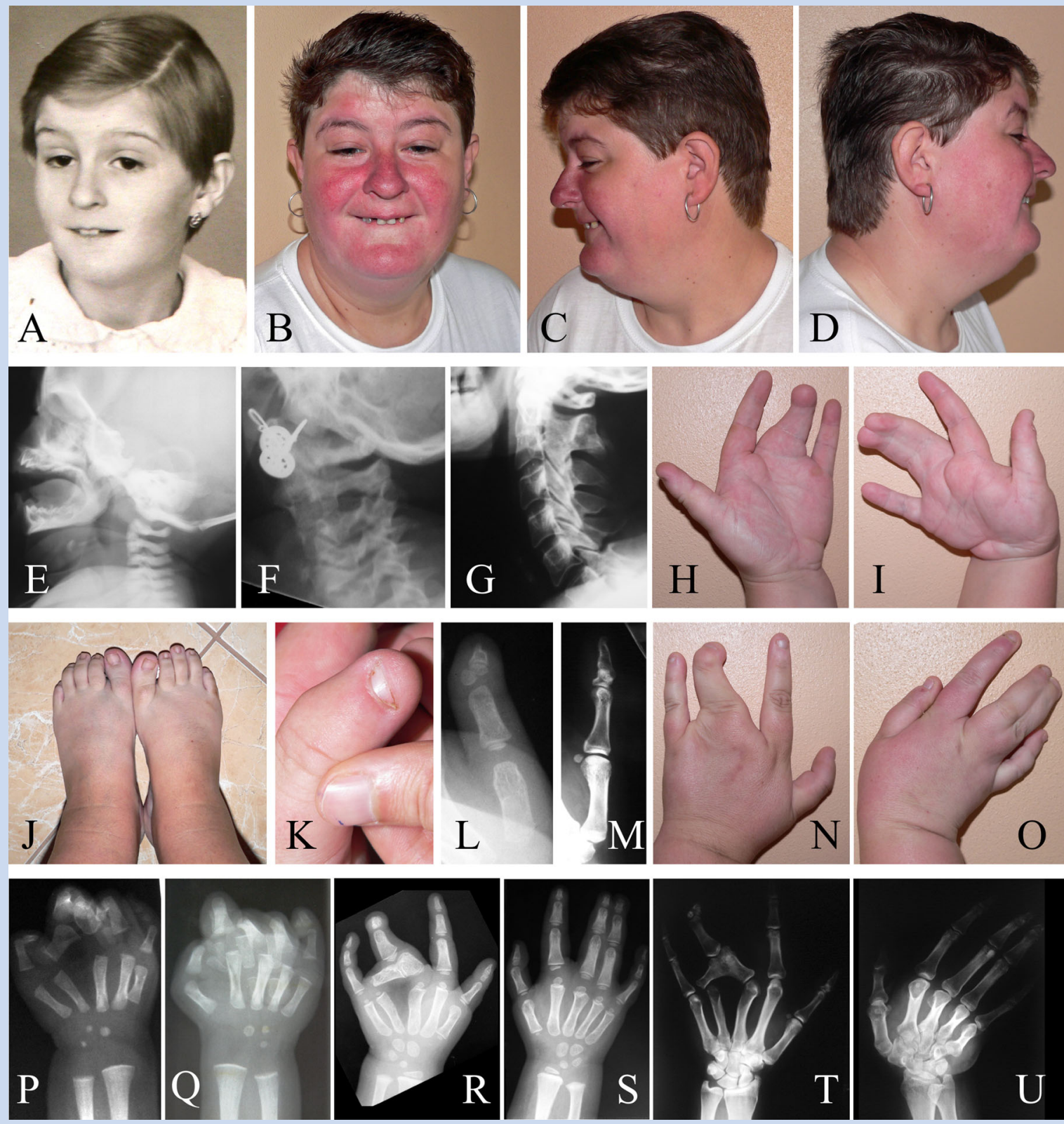

FIG. 4. Youngest sister. Photographs at 7 years $(A)$ and 31 years of age $(B, C, D, H, I, J, K, N, 0)$. Note resemblance in facial characteristics with those in her sisters. Cervical radiographs at 2 weeks [E], 22 months [F] and 32 years $[G]$ of age, demonstrating progressive $C 2-C 3$ fusion. Left thumb at 31 years of age $(K)$ and its radiograph at 22 months $(L)$ and 32 years $(M)$ showing the described extra bone and small distal phalange. Hands' $x$-rays radiographs at 2 weeks $(P, Q), 22$ months $(R, S)$ and 32 years of age $(T, U)$. There is syndactyly between the 3 rd-4th fingers, cutaneous at the right side and osseous at the left side. Further note the advanced bone age in childhood and the shortening of the right metacarpals.

et al. [2011], but in each of these the diagnosis could be refuted because of obvious other characteristics not present in the sisters reported here.

In conclusion we described three sisters who resemble one another in their facial characteristics and intellectual disability, and also in having hand manifestations although these varied widely. Pattern of inheritance is most likely autosomal recessive although autosomal dominant with variable expression cannot be completely excluded. Further molecular studies are in progress. In addition we hope the present report will lead to the description of further cases which should allow further delineation of this entity and clarify its etiology. 


\section{ACKNOWLEDGMENTS}

We are pleased to thank the family for their cooperation. S.B.S. acknowledges a grant from Fundação para a Ciência e Tecnologia (SFRH/BD/46778/2008).

\section{REFERENCES}

Feingold M, Hall BD, Lacassie Y, Martinez-Frias ML. 1997. Syndrome of microcephaly, facial and hand abnormalities, tracheoesophageal fistula, duodenal atresia, and developmental delay. Am J Med Genet 69:245-249.

Filippi G. 1985. Unusual facial appearance, microcephaly, growth and mental retardation, and syndactyly. A new syndrome? Am J Med Genet 22:821-824.

Gorlin RJ, Meskin LH, Geme JW. 1963. Oculodentodigital dysplasia. J Pediatr 63:69-75.
Hennekam RC, Krantz I, Allanson J. 2010. Gorlin's syndromes of the head and neck. 5th edition. New York: Oxford University Press.

Monfort S, Blesa D, Roselló M, Orellana C, Oltra S, Cruz Cigudosa J, Martinez F. 2007. Duplication of 14q11.2 associates with short stature and mild mental retardation: A putative relation with quantitative trait loci. Am J Med Genet Part A 384A:382-384.

Ravel A, Chouery E, Stora S, Jalkh N, Villard L, Temtamy S, Mégarbané A. 2011. How many entities exist for the spectrum of disorders associated with brachydactyly, syndactyly, short stature, microcephaly, and intellectual disability? Am J Med Genet Part A 155A:880-884.

Tonoki H, Kishino T, Niikawa N. 1990. A new syndrome of dwarfism, brachydactyly, nail dysplasia, and mental retardation in sibs. Am J Med Genet 36:89-93.

Tsukahara M, Azuno Y, Kajii T. 1989. Type A1 brachydactyly, dwarfism, ptosis, mixed partial hearing loss, microcephaly, and mental retardation. Am J Med Genet 33:7-9. 